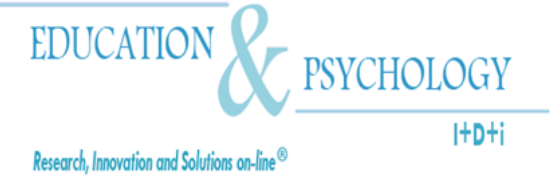

\title{
Metas Académicas: Perspectiva Histórica y Conceptual e Implicaciones Educativas
}

\section{Antonio Valle ${ }^{1}$, Susana Rodríguez ${ }^{1}$, Ramón G. Cabanach ${ }^{1}$, José C. Núñez ${ }^{2}$, Julio A. González-Pienda ${ }^{2}$, y Pedro Rosario ${ }^{3}$ \\ ${ }^{1}$ Dpto. de Psicología Evolutiva y de la Educación, Universidad de A Coruña ${ }^{2}$ Departamento de Psicología, Universidad de Oviedo ${ }^{3}$ Departamento de Psicología de la Educación, Universidad de Minho, Portugal}

\section{España / Portugal}

Correspondencia: Antonio Valle Arias. Universidad de A Coruña, Departamento de Psicología Evolutiva y de la Educación. Campus de Elviña s/n, 15071 A Coruña. Spain. E-mail: vallar@udc.es

(C) Education \& Psychology I+D+i and Editorial EOS (Spain) 


\title{
Resumen
}

El objetivo de este trabajo es ofrecer un panorama histórico y conceptual de la teoría de las metas de logro. El punto de partida es la diferenciación entre metas de aprendizaje y metas de rendimiento. Posteriormente se establece la distinción entre tendencias de aproximación y de evitación, primero en las metas de rendimiento y luego también en las metas de aprendizaje. Finalmente surge la perspectiva de las múltiples metas que contempla combinaciones de varias metas que dan lugar a diferentes perfiles motivacionales. El enfoque de las múltiples metas constituye una línea de investigación mucho más próxima y ajustada al funcionamiento real de la motivación en contextos académicos.

Palabras clave: metas de aprendizaje, metas de rendimiento, metas de aproximación, metas de evitación, múltiples metas.

Recibido: 09/03/09 Aceptación inicial: 13/03/09 Aceptación final: 01/09/09

\section{Academic Goals: Historical and Conceptual Perspectives and Educational Implications}

\begin{abstract}
The aim of this work is to provide a historical and conceptual overview of achievement goals. The starting point is the learning and performance goals differentiation. Later approach and avoidance regulation was established, first on performance goals and then also on learning goals. Finally, multiple goals perspective as a multiple goals combinations that leading to different motivational profiles arises. The multiple goals approach represents an online research next and adjusted to actual functioning of motivation in academic contexts.
\end{abstract}

Keywords: learning goals, performance goals, approach goals, avoidance goals, multiple goals.

Received: 03/09/09 Initial Acceptance: 03/13/09 Definitive Acceptance: 09/01/09

1074 - 


\section{Introducción}

Las metas personales constituyen un marco de referencia de importancia incuestionable para explicar las orientaciones motivacionales y los patrones de comportamiento en el ámbito escolar. Aunque se ha abordado su estudio desde diferentes perspectivas, todas ellas comparten la idea de que las personas establecen metas para sí mismas, de tal forma que estas representaciones cognitivas de los eventos futuros se convierten en potenciales motivadores de la conducta en cualquier contexto (Tapola y Niemivirta, 2008; Was, Al-Harthy, StackOden y Isaacson, 2009). Bajo estos planteamientos, y dentro del ámbito académico, ha cobrado un especial interés la noción de que las metas académicas que persigue el estudiante organizan y regulan su comportamiento de cara a la consecución de un determinado logro, estrechamente relacionado con el tipo de motivación definido por la clase de meta que se pretende conseguir (Valle, Núñez, Cabanach, Rodríguez, González-Pienda y Rosario, 2009).

A la propuesta inicial de los primeros autores (véase p.e., Dweck, 1986; Nicholls, 1984), que sólo distinguían entre metas de aprendizaje y metas de rendimiento, le siguió el enfoque tridimensional (Elliot, 1997, Elliot y Harackiewicz, 1996; Middleton y Midgley, 1997; Phan, 2008; Skaalvik, 1997), que incluía la diferenciación entre tendencias de aproximación y de evitación en las metas de rendimiento (Bartels, Magun-Jackson y Kemp, 2009), y luego los modelos 2x2 (Elliot, 1999; Elliot y McGregor, 2001; Pintrich, 2000a, 2000b) que distinguían cuatro tipos de metas al contemplar las tendencias de aproximación y de evitación dentro de las metas de aprendizaje y de las metas de rendimiento. Posteriormente, también fueron surgiendo algunas ampliaciones de la teoría de metas al incorporar otros tipos de metas con un carácter más social (véase p.e., Wentzel, 2000, 2001). Sin embargo, por su carácter integrador, el enfoque de las múltiples metas es, probablemente, una de las propuestas más novedosas y de mayor relevancia educativa en la reciente historia de la investigación sobre metas académicas. Por tanto, desde los trabajos pioneros del enfoque bidimensional hasta la actualidad, la teoría de las metas de logro ha sufrido modificaciones importantes (ver Tabla $1)$.

De todas formas, aunque los trabajos iniciales del enfoque bidimensional contribuyeron a desarrollar una importante línea de investigación con gran proyección de cara al futuro, sí es necesario reconocer que estos primeros trabajos sólo permitieron contemplar formas de 
aproximación a la motivación, dejando de lado la distinción aproximación-evitación. Esto resulta bastante incomprensible si se tiene en cuenta que la diferencia entre aproximación y evitación ya aparecía incluida dentro de los modelos clásicos sobre la motivación de logro. De hecho, tanto McClelland (1951) como Atkinson (1957) ya establecían la diferencia entre deseo de obtener el éxito y deseo de evitar el fracaso.

Tabla 1. Evolución de la teoría de las metas de logro

ENFOQUE BIDIMENSIONAL: (Dweck, 1986; Nicholls, 1984): Metas de aprendizaje versus metas de rendimiento

ENFOQUE TRIDIMENSIONAL (Elliot, 1997, Elliot y Harackiewicz, 1996; Middleton y Midgley, 1997; Skaalvik, 1997): Metas de aprendizaje, metas de aproximación al rendimiento, metas de evitación del rendimiento.

MODELO 2x2 (Elliot, 1999; Elliot y McGregor, 2001; Pintrich, 2000a, 2000b):

Diferenciación entre orientación de meta (aprendizaje versus rendimiento) y dirección de la meta (aproximación versus evitación).

Actualmente, todos los teóricos de la motivación consideran que la conducta motivada puede estar dirigida por posibilidades positivas o negativas. Todas las metas están focalizadas en cualquier posibilidad positiva o negativa y tienen una función direccional en la motivación, guiando al individuo hacia resultados o estados positivos potenciales o alejándolo de resultados o estados negativos potenciales (Elliot y Church, 1997). Por tanto, una tendencia de aproximación lleva a las personas a moverse hacia estados finales positivos o deseados intentando que se produzcan, mientras que una tendencia de evitación lleva a las personas a alejarse de estados finales negativos o no deseados intentando que no se produzcan.

Esta diferenciación entre metas de aproximación y metas de evitación (dirección de la meta) se aplica a las metas de aprendizaje y a las metas de rendimiento (orientación de la meta) dando lugar a los cuatro tipos de metas propuestos por el modelo $2 \times 2$. 


\section{El enfoque bidimensional: Metas de aprendizaje versus metas de rendimiento}

En la literatura contemporánea sobre la motivación de logro, el enfoque de las metas académicas planteado inicialmente por Dweck (1986), Nicholls (1984) y otros autores (p.e., Ames, 1984) se ha convertido en una de las principales líneas de investigación, dada su relevancia sobre la cognición, el comportamiento y el afecto.

Aunque los motivos que guían la conducta son diversos, la mayoría de los estudiantes suelen implicarse en las actividades o trabajos que requieren dedicación y esfuerzo por dos tipos de razones diferentes. Por un lado, están aquellas que tienen que ver con dar o mantener una imagen, alcanzar un resultado o mostrar la capacidad de uno mismo, habitualmente denominadas como metas de rendimiento o de ejecución y, por otro, están las llamadas metas de aprendizaje o de dominio, que tienen más que ver con razones vinculadas al trabajo o la actividad en sí misma, relacionándose con su disfrute o su dominio.

Considerando esta diferenciación, los individuos pueden estar implicándose en una actividad debido a su deseo de aprender, desarrollar o mejorar sus capacidades o bien con objeto de demostrar su capacidad o de proteger su imagen personal buscando las valoraciones positivas de los demás. Concretamente, en el ámbito educativo se ha diferenciado entre estudiantes que sostienen metas de aprendizaje para incrementar su capacidad y aquellos que optan por metas de rendimiento para demostrar su capacidad (Elliot, 1999; Pajares, Britner y Valiante, 2000).

La mayoría de las investigaciones sobre las metas académicas se han centrado en estos dos tipos de metas: metas de rendimiento (también denominadas metas de ejecución o metas centradas en el yo), que se focalizan en la demostración de la competencia respecto a otros, y las metas de aprendizaje (también denominadas metas de de dominio o metas centradas en la tarea), que se centran en el desarrollo de la competencia y el dominio de las tareas (ver Figura $1)$. 
METAS DE APRENDIZAJE

[de dominio o centradas en la tarea]

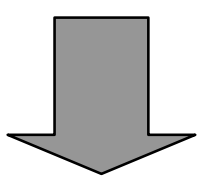

Objetivo:

INCREMENTAR

LA CAPACIDAD
METAS DE RENDIMIENTO

[de ejecución o centradas en el yo]

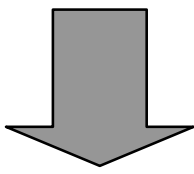

Objetivo:

DEMOSTRAR LA

CAPACIDAD

Figura 1. Metas de aprendizaje versus metas de rendimiento

A la luz de los conocimientos derivados de la investigación en torno a las metas de logro se considera que la preferencia por unos motivos u otros conlleva un patrón peculiar de comportamientos y resultados (Valle et al., 2009b). Detrás de una orientación general al aprendizaje o al dominio nos encontramos con personas que buscan la excelencia en el trabajo, que sienten que realmente eligen las cosas que hacen y que, además, éstas les interesan. Los individuos que muestran una preferencia por motivos de dominio suelen estar realmente interesados en las actividades o tareas que abordan y esto les lleva a dedicar esfuerzo y a persistir ante cualquier dificultad. Posiblemente no sean los más eficientes para tareas rutinarias pero con el tiempo acaban dominando todos los entresijos en las áreas en las que trabajan.

Por otro lado, la especial preocupación que tienen algunas personas por la imagen y el valor otorgado a la competitividad puede llevarlas a implicarse en el trabajo con objeto tanto de mostrar sus capacidades como de evitar que se conozcan sus limitaciones. Hacerlo mejor que los demás o resolver problemas y situaciones que otros no pueden se convierten, de este modo, en poderosos motivadores de la acción para muchos individuos. Del mismo modo, algunas personas pueden verse atraídas hacia la acción procurando que los demás no se enteren de que tienen dificultades, con objeto de evitar burlas o por no perder el respeto de referentes importantes para ellas. Si bien están vinculadas con la imagen y el rendimiento, este último tipo de razones para la implicación tienen un carácter más defensivo o de protección. Es posible que las personas preocupadas por su imagen, buscando sacar provecho de sus es- 
fuerzos, se ajusten a las reglas y sean relativamente eficaces en labores técnicas o que requieran una dedicación más superficial.

Las mismas preocupaciones a las que nos referíamos como activadoras de la actuación pueden funcionar como inhibidoras de la acción. Así, por ejemplo, la preocupación por la imagen personal que lleva a algunos individuos a implicarse en el trabajo, puede llevarles a evitar el compromiso cuando esa preocupación se expresa en los términos siguientes: "evito trabajar si veo que seré el que peor lo haga", "no suelo comprometerme en trabajos si creo que no voy a obtener los mejores resultados del grupo", o "si veo que voy a fracasar en una prueba, ya no me esfuerzo en intentarlo".

Los individuos que mantiene este tipo de preocupaciones no sólo evitan la dedicación al estudio o al trabajo sino que pueden estar haciendo grandes esfuerzos por ganarse de modos singulares el afecto de los demás. De hecho, es probable que muchas personas con baja autoestima hayan aprendido a "sobrevivir" en la sociedad actual refugiándose tras cualquier estratagema que les permita evitar riesgos para su autovalía. Así, podemos encontrarnos con individuos que prefieren presentarse como poco hábiles, que suelen mostrarse ante los demás pesimistas sobre sus resultados, al margen de sus posibilidades reales, o que encuentran todo tipo de excusas o explicaciones con tal de no tener que arriesgar su valía personal (Rodríguez, Cabanach, Valle, Núñez, y González-Pienda, 2004; Valle, Cabanach, Rodríguez, Núñez y González-Pienda, 2005; Valle, Núñez, Cabanach, Rodríguez, González-Pienda y Rosário, 2007).

El enfoque tridimensional: Metas de aprendizaje, metas de aproximación al rendimiento y metas de evitación del rendimiento

Revisando la dicotomía aprendizaje-rendimiento, Elliot y sus colegas (Elliot, 1997; Elliot y Church, 1997; Elliot y Harackiewicz, 1996) han propuesto un marco tridimensional para las metas académicas. En esta propuesta, el constructo metas de rendimiento se diferencia en dos formas de regulación: una de aproximación y otra de evitación, delimitándose, por tanto, tres metas académicas independientes: unas metas de aproximación al rendimiento (performance-approach), focalizadas en el logro de competencia con relación a otros; unas metas de evitación del rendimiento (performance-avoidance), centradas en la evitación de 
incompetencia respecto a otros (ver Figura 2); y unas metas de aprendizaje, centradas en el desarrollo de la competencia y el dominio de la tarea.

Cada una de estas metas lleva a un patrón exclusivo de procesos de logro y resultados. Concretamente, en algunos de los estudios llevados a cabo por estos autores se indica que las metas de aprendizaje están relacionadas positivamente con el interés, las metas de aproximación al rendimiento muestran relaciones positivas con el rendimiento académico, y las metas de evitación del rendimiento se relacionan negativamente con el interés y con el rendimiento. En la misma línea, Elliot, McGregor y Gable (1999), encontraron que las metas de aprendizaje predicen positivamente la persistencia, el esfuerzo y el procesamiento profundo; las metas de aproximación al rendimiento predicen positivamente el procesamiento superficial, la persistencia, el esfuerzo y el rendimiento en los exámenes; y las metas de evitación del rendimiento predicen positivamente el procesamiento superficial y negativamente el rendimiento y el procesamiento profundo.

Figura 2. Metas de aprendizaje, metas de aproximación al rendimiento y metas de evitación del rendimiento

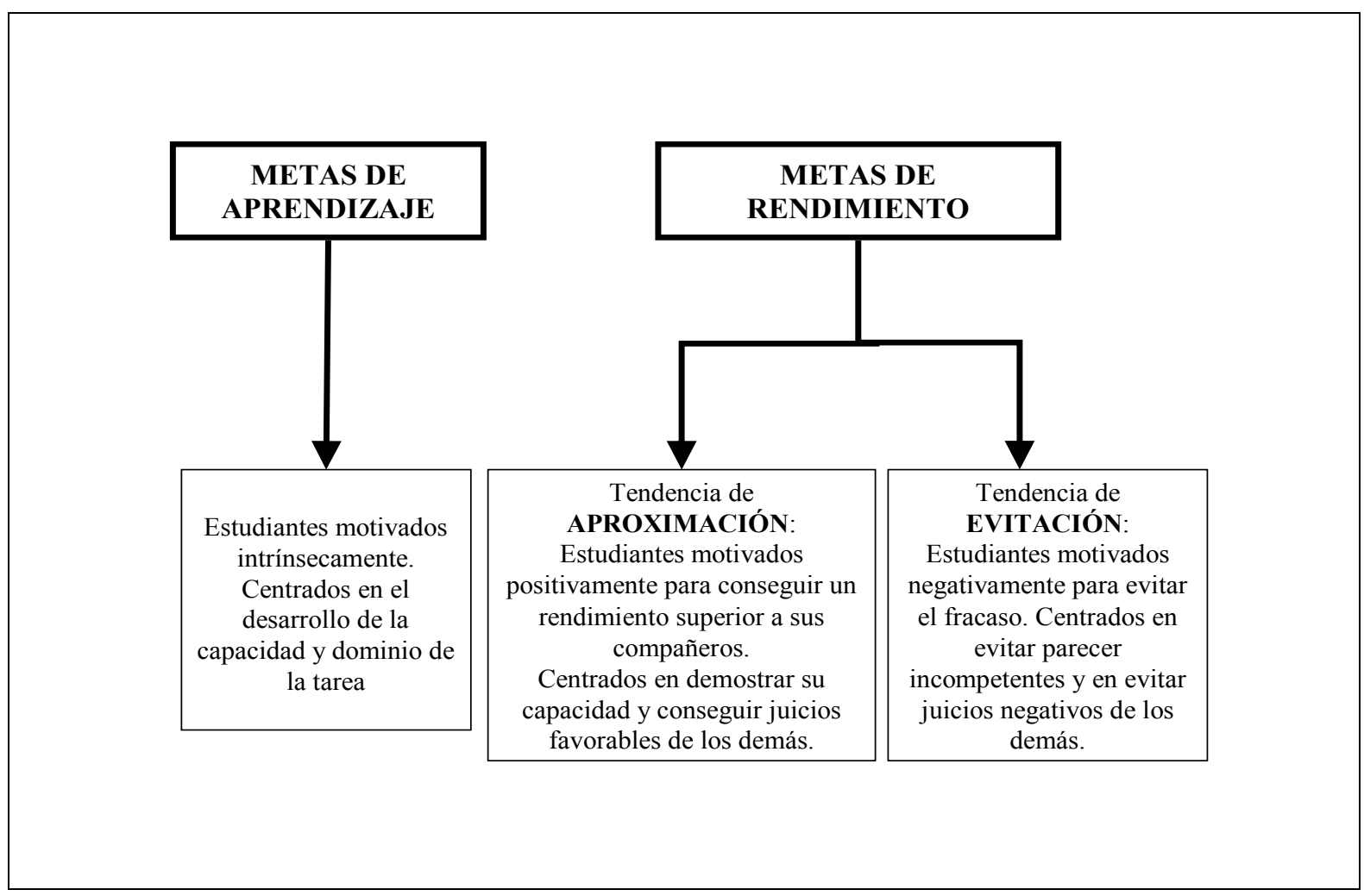

Diferentes estudios han proporcionado hallazgos empíricos que ofrecen un respaldo a la distinción entre tendencias de aproximación y tendencias de evitación dentro de las metas 
de rendimiento (véase, por ejemplo, Elliot y Church, 1997; Elliot y Harackiewicz, 1996; Middleton y Midgley, 1997; Rodríguez, Cabanach, Piñeiro, Valle, Núñez y González- Pienda, 2001; Valle, Cabanach, Rodríguez, Núñez y González-Pienda, 2006; Wolters, Yu y Pintrich, 1996). Además, la diferenciación entre metas de aproximación al rendimiento y metas de evitación del rendimiento ha llevado a los expertos a reconsiderar los efectos perjudiciales de las metas de aproximación al rendimiento. Así, Pintrich (2000c) señala que pueden darse situaciones en que las metas de rendimiento no sean desadaptativas en términos motivacionales, afectivos, de uso de estrategias y de rendimiento. De hecho, en algunos trabajos las metas de aproximación al rendimiento aparecen más vinculadas al rendimiento y al logro, mientras que las metas de aprendizaje lo están con un mayor interés intrínseco en las tareas (veáse, por ejemplo, Harackiewicz, Barron, Pintrich, Elliot y Thrash, 2002)

En todo caso, los diferentes propósitos para el compromiso con la tarea se han contemplado como predictores importantes de un buen número de procesos y de resultados. Del mismo modo que consideramos relevante profundizar en la divergencia de los resultados obtenidos al comparar las metas de aprendizaje y las metas de aproximación al rendimiento, también creemos importante resaltar las diferencias entre la tendencia de aproximación y la tendencia de evitación entre las metas de rendimiento. De hecho, estas dos aproximaciones podrían presentan un patrón más divergente de resultados que el obtenido para las metas de aprendizaje y de aproximación al rendimiento.

Cuando el criterio mediante el cuál el estudiante estima sus progresos se refiere a los demás, y la principal preocupación es no estar entre los peores, nos encontramos con un patrón de actuación que difiere sustancialmente de una orientación de aproximación al rendimiento. La dedicación de esfuerzo tiende a evitarse y el abandono ante pequeñas dificultades es un hecho. Las tareas complicadas se perciben como una amenaza y el aula puede convertirse fácilmente en un tormento. En la Tabla 2 se resumen algunas de las diferencias entre los estudiantes que adoptan los distintos tipos de metas propuestas por el enfoque tridimensional. 
Tabla 2. Algunas diferencias entre los estudiantes que adoptan distintos tipos de metas (Cabanach Valle, Rodríguez, Piñeiro y García , 2007, p. 44)

\begin{tabular}{|c|c|c|c|}
\hline & M. APREND. & M. APROX. RENDIM. & M. EVIT. RENDIM. \\
\hline $\begin{array}{l}\text { Principal } \\
\text { preocupación del } \\
\text { estudiante }\end{array}$ & “APRENDER” & "SER EL MEJOR" & "NO SER EL PEOR" \\
\hline $\begin{array}{c}\text { ¿Cómo perciben las } \\
\text { tareas? }\end{array}$ & $\begin{array}{l}\text { "A VER SI LA DOMINO" } \\
\text { (RETO) }\end{array}$ & $\begin{array}{l}\text { “A VER SI SOY EL MEJOR” } \\
\text { (RETO) }\end{array}$ & $\begin{array}{l}\text { "QUÉ DESASTRE SI ME } \\
\text { SALE MAL" (AMENAZA) }\end{array}$ \\
\hline $\begin{array}{l}\text { Las tareas difíciles } \\
\text { son vistas como... }\end{array}$ & $\begin{array}{l}\text { UNA OPORTUNIDAD } \\
\text { PARA APRENDER }\end{array}$ & $\begin{array}{l}\text { UNA OPORTUNIDAD PARA } \\
\text { DESTACAR }\end{array}$ & UNA AMENAZA \\
\hline Los errores son... & $\begin{array}{l}\text { ALGO NATURAL, } \\
\text { PARTE DEL PROCESO } \\
\text { DE APRENDIZAJE }\end{array}$ & $\begin{array}{l}\text { UN FRACASO, UNA } \\
\text { PRUEBA DE FALTA DE } \\
\text { CAPACIDAD }\end{array}$ & $\begin{array}{c}\text { UN FRACASO, UNA } \\
\text { PRUEBA DE FALTA DE } \\
\text { CAPACIDAD }\end{array}$ \\
\hline $\begin{array}{c}\text { Su persistencia en las } \\
\text { tareas es... }\end{array}$ & ALTA & ALTA & BAJA \\
\hline ¿Se esfuerzan? & $\begin{array}{c}\text { Sí, SE ESFUERZAN POR } \\
\text { APRENDER }\end{array}$ & UTILIZAN EL ESFUERZO & EVITAN EL ESFUERZO \\
\hline $\begin{array}{l}\text { ¿Cómo evalúan su } \\
\text { propia actuación? }\end{array}$ & $\begin{array}{l}\text { CRITERIOS DE ÉXITO } \\
\text { AUTORREFERIDOS }\end{array}$ & $\begin{array}{l}\text { SE COMPARAN CON LOS } \\
\text { DEMÁS: "SACAR LAS } \\
\text { MEJORES NOTAS, SER EL } \\
\text { MEJOR DE LA CLASE..." }\end{array}$ & $\begin{array}{l}\text { SE COMPARAN CON } \\
\text { LOS DEMÁS: "NO } \\
\text { SACAR LAS PEORES } \\
\text { NOTAS, NO SER EL } \\
\text { PEOR DE LA CLASE..." }\end{array}$ \\
\hline
\end{tabular}

\section{El modelo 2x2: La diferenciación entre orientación y dirección de las metas}

Los modelos 2x2 (Elliot, 1999; Elliot y McGregor, 2001; Pintrich, 2000a, 2000b) suponen una ampliación del enfoque tridimensional en el sentido de que contemplan también la tendencia de aproximación dentro de las metas de aprendizaje o de dominio. Por tanto, diferencian tanto la orientación -aprendizaje versus rendimiento- como la dirección -aproximación versus evitación- de las metas. De acuerdo con este modelo, se pueden adoptar cuatro tipos de orientaciones de meta distintas: metas de aproximación al dominio o al aprendizaje, metas de evitación del dominio o del aprendizaje, metas de aproximación al rendimiento y metas de evitación del rendimiento (Harackiewicz y Linnenbrik, 2005).

Aunque la principal novedad de los modelos 2x2 fue la incorporación de las metas de evitación del aprendizaje, hay que señalar que todavía están bastante indefinidas dentro de las investigaciones realizadas en el campo de las metas académicas. En un intento de mantener un cierto paralelismo con los otros tres tipos de metas, la tendencia de evitación en las metas de aprendizaje estaría orientada a evitar el "no dominio" o "el no aprendizaje o comprensión" de las tareas. Los estándares que se usarían en esta orientación reflejarían una preocupación por no aprender y por cometer errores de comprensión, pero no con respecto a otros sino en referencia a uno mismo o a la propia tarea (estándar intrapersonal o absoluto). El no aprender o el 
cometer errores de comprensión puede llevar efectivamente a una perdida de confianza en uno mismo y/o a una excesiva preocupación por no hacerlo mal con respecto a ellos mismos y a sus propios y exigentes criterios. Nos referiríamos en este caso a individuos extremadamente exigentes y perfeccionistas que rara vez se sienten a gusto después de haber realizado una tarea o actividad y/o sujetos que no muestran disposición a implicarse en actividades que no satisfacen sus propios intereses intrínsecos o disfrute personal. Al menos teóricamente podemos sugerir algunos ítems a utilizar para evaluar este tipo de metas: "Me esfuerzo porque me preocupa no aprender en esta clase todo lo que podría", "Me esfuerzo porque temo no llegar comprender el contenido de esta clase tan a fondo como me gustaría", "Me esfuerzo porque me preocupa no aprender en esta clase todo lo que hay que aprender".

Partiendo de la idea de que las metas de logro adoptadas para guiar la actuación son, de algún modo, representaciones cognitivas de propias posibilidades positivas o negativas, en términos de competencia, estas creencias de competencia o autoeficacia se han considerado un punto central de la teoría de las metas de logro desde sus inicios hasta la actualidad. Elliot y McGregor (2001) observaron la competencia en base a dos dimensiones fundamentales: cómo es definida y cuál es su valencia. Así, la competencia podría definirse en función del estándar utilizado para la evaluación de la actuación, estándar que puede ser absoluto -los criterios son los requisitos de la tarea en sí misma-, intrapersonal -el criterio es el logro pasado o el logro potencial de uno mismo- y normativo -el criterio es la ejecución de otros-. La otra dimensión definitoria de la competencia: la valencia puede referirse o bien una posibilidad deseable y positiva (p.e., éxito) o bien a una opción no deseable y negativa (p.e., fracaso). Desde esta perspectiva no sería posible formular una meta sin tomar en consideración, implícita o explícitamente, información referida a la definición de competencia y a la valencia. (Elliot y McGregor, 2001).

En base a estos parámetros cabe diferenciar tipos de metas (ver Tabla 3): (a) Las metas de aproximación al aprendizaje, en las que la competencia se define en términos absolutos/intrapersonales y su valencia es positiva; (b) las metas de aproximación al rendimiento, en las que la competencia se define normativamente y su valencia es positiva; (c) las metas de evitación del aprendizaje, en las que la competencia se define en términos absolutos/intrapersonales y su valencia es negativa; y (d) las metas de evitación del rendimiento, en las que la competencia se define normativamente y su valencia es negativa. 
Tabla 3. Tipos de metas según el modelo 2x2 (Elliot y McGregor, 2001, p. 502)

Definición

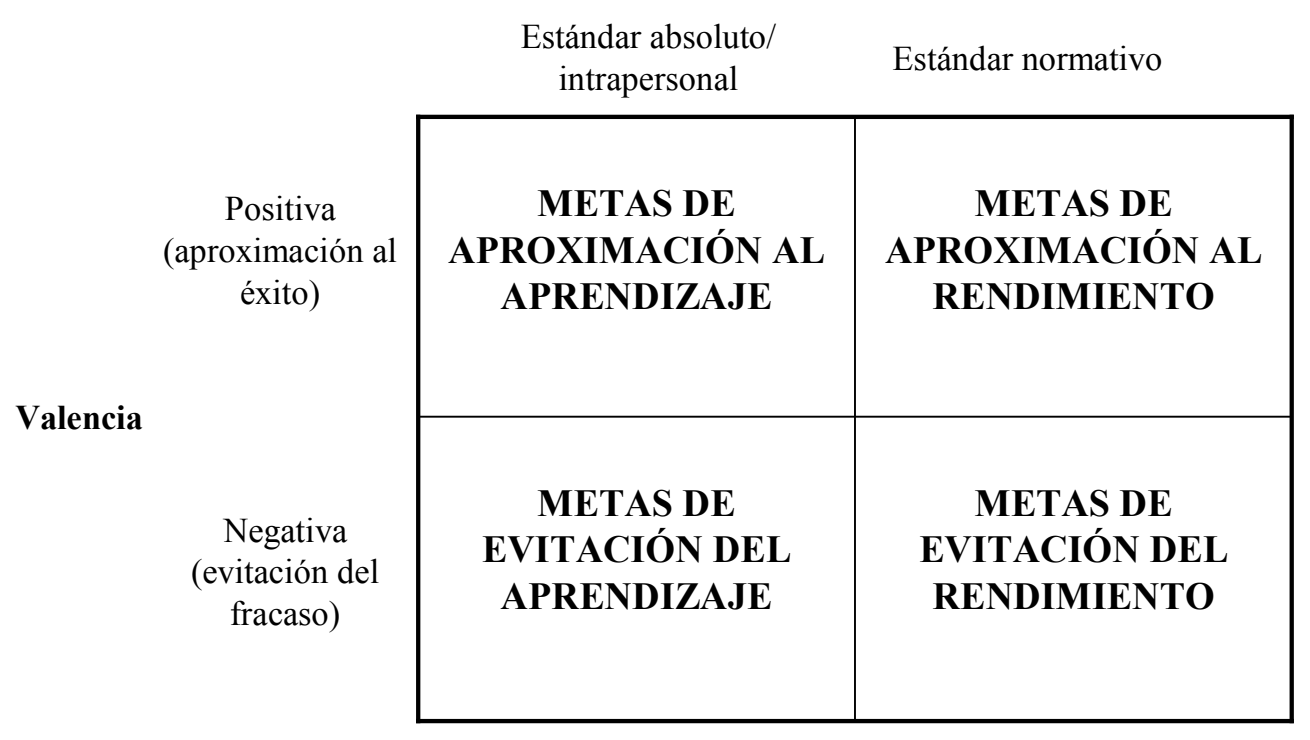

\section{Combinación de metas y perfiles motivacionales: El enfoque de las múltiples metas}

La posibilidad de que metas tradicionalmente conceptualizadas de un modo excluyente -metas de aprendizaje vs. metas de rendimiento- puedan complementarse se viene defendiendo en los últimos años como una manera más integradora y realista de abordar el estudio de las metas académicas. Esta perspectiva de trabajo que se ha referido como el enfoque de las múltiples metas es relativamente reciente dentro la investigación en el campo de las metas académicas y postula que en la realidad del aula los estudiantes pueden perseguir metas de aprendizaje y de rendimiento al mismo tiempo, utilizando unas u otras en función de sus características personales, de la tarea y/o de variables situacionales y contextuales.

A este respecto, Barron y Harackiewicz (2001) han sugerido cuatro modos mediante los cuales las metas de aprendizaje y las metas de aproximación al rendimiento podrían combinarse. Una primera vía sería aquella en la que ambos tipos de metas se combinan pero cada una tiene efectos beneficiosos para determinados resultados, esto es, sus efectos son aditivos. Una segunda posibilidad es que se den efectos interactivos entre los dos tipos de metas, de modo que adoptar ambas metas resulte más adaptativo para un determinado resultado que adoptar un único tipo. La tercera opción es que se den efectos especializados, esto es, que haya unos efectos únicos para ambos tipos de metas a lo largo de múltiples resultados (por 
ejemplo, que las metas de aprendizaje sean beneficiosas en términos de interés o bienestar emocional mientras que las metas de aproximación al rendimiento resulten adaptativas para resultados tales como el logro). Y por último, existe la posibilidad de que se den efectos selectivos, de manera que las consecuencias o efectos de las metas dependan de si coinciden o no con las metas del contexto.

A nivel empírico, Pintrich y Garcia (1991), utilizando el análisis de conglomerados con una muestra de estudiantes universitarios, encontraron que el grupo con elevadas metas de aprendizaje y bajas metas de rendimiento evidenciarían un patrón motivacional más adaptativo que otras combinaciones de metas. Estos autores constataron también que en ausencia de metas de aprendizaje, sería la preocupación por el rendimiento la que podría estar motivando a los estudiantes a implicarse, en alguna medida, en las actividades de estudio. De modo similar, Meece y Holt (1993) observaron que el grupo de estudiantes de primaria con altas metas de aprendizaje y bajas metas de rendimiento presentaba un patrón más adaptativo que otras combinaciones en lo que respecta al uso de estrategias cognitivas y al rendimiento real. Por su parte, Wolters et al. (1996) no hallaron interacciones significativas entre los dos tipos de metas, y las pocas interacciones que salieron a la luz se situaban en la línea de lo defendido por la teoría normativa de metas: el grupo con elevada orientación al aprendizaje y baja orientación al rendimiento podría ser más adaptativo en términos de autoeficacia, valor de la tarea y uso de estrategias cognitivas y metacognitivas.

Entre los estudios que han encontrado que los patrones óptimos se vinculan a perfiles con niveles elevados en ambos tipos de metas está el de Bouffard, Boisvert, Vezeau y Larouche (1995), cuyos resultados demuestran que los niveles más elevados de motivación, uso de estrategias cognitivas, autorregulación y rendimiento aparecen en el grupo que combina elevados niveles de metas de aprendizaje y de rendimiento, seguido éste por el grupo con altas metas de aprendizaje y bajas metas de rendimiento. Por su parte, Harackiewicz, Barron, Tauer, Carter y Elliot (2000) al tratar de comprobar las consecuencias a corto y largo plazo de las metas de logro, se encontraron con que las metas de aprendizaje predecían positivamente el interés pero no las calificaciones, mientras que las metas de aproximación al rendimiento sí predecían las calificaciones pero no el interés.

Este conjunto de resultados ha llevado a hipotetizar que ambos tipos de metas pueden tener efectos beneficiosos en contextos académicos y, como consecuencia, la perspectiva teó- 
rica de las múltiples metas pasa a convertirse en una potente alternativa para la explicación motivacional de gran interés para los estudiantes. Pintrich (2000c), en un estudio longitudinal realizado con estudiantes de secundaria, concluye que los alumnos preocupados por su rendimiento y por ser mejores que sus compañeros, pero que al mismo tiempo están orientados al aprendizaje siguen una trayectoria paralela a aquellos que caracterizados por una orientación al aprendizaje. Sin embargo, el autor también añade que esta trayectoria ya no resulta igualmente adaptativa en el caso de aquellos estudiantes únicamente preocupados por el rendimiento.

Desde la perspectiva socioconstructiva, Paris y Turner (1994) introducen el término de "motivación situada" para describir el carácter situacional y contextual de la conducta motivada, de tal forma que aunque la motivación sea fruto de un proceso de construcción interno y personal, también es evidente que está condicionada por las condiciones del contexto en el que se va a manifestar (Levy-Tossman, Kaplan y Assor, 2007). Los datos parecen evidenciar que son precisamente aquellos estudiantes con múltiples metas los que mostrarían mejores niveles de adaptación a las demandas de las diversas situaciones de aprendizaje. Los resultados obtenidos en nuestro país en el ámbito de la educación superior apoyarían también este planteamiento. Concretamente, la investigación de Cabanach, Valle, Piñeiro, Rodríguez y Núñez (1999) puso de relieve que los estudiantes con múltiples metas se adaptan mejor a las demandas contextuales, especialmente al estilo de enseñanza y al sistema de evaluación. En concreto, dependiendo del estilo de enseñanza del profesor y de los criterios de evaluación que utiliza, la forma de estudiar y la calidad del proceso de estudio va a ser diferente. Los hallazgos del trabajo de Suárez, Cabanach y Valle (2001) ponen de relieve que la adopción simultánea de las metas de tarea (metas de aprendizaje) y autoensalzamiento del ego (metas de aproximación al rendimiento) constituiría la mejor combinación de metas mejor a la hora de posibilitar que el estudiante desarrolle un control directo y positivo sobre todas y cada una de las estrategias autorreguladoras. La gestión de las orientaciones de meta le permite optimizar la gestión estratégica en respuesta a las demandas derivadas de las distintas tareas y contextos y a sus propios intereses. Los resultados obtenidos por Rodríguez et al. (2001) demuestran no sólo que la adopción de múltiples metas conduce a un mejor rendimiento académico, sino también que la coordinación de distintos tipos de metas favorece el desarrollo de habilidades de autorregulación que permiten adaptarse más eficazmente a las demandas del contexto de aprendizaje. 
Si bien no todos los estudiantes muestran esta capacidad de optar por múltiples metas, algunos ponen en juego habilidades suficientes para realizar una coordinación efectiva y conseguir la forma de lograr distintos tipos de metas. Por tanto, el hecho de que los estudiantes opten simultáneamente por varias metas en situaciones de logro concretas puede ser una de las opciones que presenta mayores beneficios a nivel académico (Cabanach et al., 1999; Rodríguez et al., 2001; Valle, Cabanach, Núñez, González-Pienda, Rodríguez y Piñeiro, 2003; Valle et al., 2009; Valle, Núñez, Rodríguez, Cabanach, González-Pienda y Rosário, en prensa), y emocional (Daniels, Haynes, Stupnisky, Perry, Newall y Pekrun, 2008).

En un trabajo reciente (Valle et al., 2009), en el que se pretendía constatar la existencia de combinaciones de metas entre los estudiantes de secundaria, se identificaron mediante el análisis de conglomerados cuatro grupos con perfiles motivacionales distintos (ver Tabla 4). Un primer grupo que se caracterizaba por un perfil motivacional orientado al aprendizaje y al logro (Grupo MAPL), un segundo grupo con un perfil motivacional con alta motivación generalizada (Grupo AMG), un tercer grupo con un perfil motivacional con predominio de miedo al fracaso (Grupo MF), y finalmente, un cuarto grupo con un perfil con baja motivación generalizada (Grupo BMG).

Además, el grupo con alta motivación generalizada y el grupo con motivación hacia el aprendizaje y al logro eran los que presentaban unos valores significativamente más altos en estrategias cognitivas y en estrategias de autorregulación del estudio. Por el contrario, el grupo con predominio de miedo al fracaso y el grupo con baja motivación generalizada fueron los que obtuvieron las puntuaciones más bajas en esas estrategias (una síntesis de estos resultados puede verse en la Tabla 4).

Por lo que se refiere al rendimiento académico, el grupo MAPL presentaba unas puntuaciones significativamente más altas que los otros grupos, mientras que el grupo con predominio de miedo al fracaso presentaba las puntuaciones más bajas. Pero lo más sorprendente fue que el grupo con alta motivación generalizada obtuvo unos niveles de rendimiento muy por debajo del grupo con motivación hacia el aprendizaje y al logro. Incluso, el grupo con baja motivación generalizada presentaba unos valores más altos que los del grupo con alta motivación generalizada -aunque las diferencias no eran significativas-. Lo más probable es que el grupo con alta motivación generalizada, que presentaba los niveles más altos en las 
metas vinculadas a la propia imagen, fuera también el más proclive a realizar frecuentes valoraciones de su actuación en relación con los demás, lo cual puede generar unos altos niveles de ansiedad que suelen tener consecuencias negativas sobre el rendimiento. Por otro lado, el grupo con baja motivación generalizada era el que tenía los niveles más bajos en las metas vinculadas a la propia imagen, con lo cual se enfrentaba a situaciones donde los resultados no suponían ningún tipo de valoración de uno mismo ni de sus capacidades, lo que llevó a que sus niveles de ansiedad fueran bajos y las consecuencias sobre el rendimiento resultaran más positivas que las del grupo con alta motivación generalizada (Valle et al., 2009).

Tabla 4. Perfiles motivacionales en estudiantes de secundaria $(N=524)$

(Valle et al., 2009)

\begin{tabular}{|c|c|c|c|c|}
\hline & $\begin{array}{c}\text { Grupo MAPL } \\
\text { [27,7\%] } \\
33,4 \% \text { mujeres } \\
22,9 \% \text { hombres }\end{array}$ & $\begin{array}{c}\text { Grupo AMG } \\
\text { [23,5\%] } \\
25,4 \% \text { mujeres } \\
21,8 \% \text { hombres }\end{array}$ & $\begin{array}{c}\text { Grupo MF } \\
\text { [24,2\%] } \\
18,3 \% \text { mujeres } \\
29,2 \% \text { hombres }\end{array}$ & $\begin{array}{c}\text { Grupo BMG } \\
\qquad[\mathbf{2 4 , 6 \% ]} \\
22,9 \% \text { mujeres } \\
26,1 \% \text { hombres }\end{array}$ \\
\hline $\begin{array}{l}\text { Estrategias } \\
\text { cognitivas }\end{array}$ & + & + & - & - \\
\hline $\begin{array}{l}\text { Estrategias de } \\
\text { autorregulación }\end{array}$ & + & + & - & - \\
\hline $\begin{array}{l}\text { Rendimiento } \\
\text { académico }\end{array}$ & + & & - & \\
\hline \multicolumn{5}{|c|}{$\begin{array}{l}\text { Grupo MAPL: orientado al aprendizaje } \\
\text { predominio de miedo al fracaso; Grupo B } \\
+=\text { puntuaciones altas en la variable } \\
-=\text { puntuaciones bajas en la variable }\end{array}$} \\
\hline
\end{tabular}

Siguiendo en la línea de las múltiples metas, en otro trabajo reciente (Valle et al., en prensa) con una muestra de 1924 estudiantes universitarios, se identificaron seis perfiles motivacionales distintos (ver Tabla 5). Un primer grupo con un perfil de baja motivación generalizada (Grupo BMG), un segundo grupo con un perfil motivacional orientado a evitar dar una mala imagen ante los demás (Grupo MER), un tercer grupo con un perfil motivacional orientado al aprendizaje (Grupo MAP), un cuarto grupo con un perfil motivacional orientado al aprendizaje y a evitar dar una mala imagen ante los demás (Grupo MAP/MER), un quinto 
Tabla 5. Perfiles motivacionales en estudiantes universitarios ( $N=1924)$ (Valle et al., en prensa)

\begin{tabular}{|c|c|c|c|c|c|c|}
\hline & $\begin{array}{c}\text { Grupo BMG } \\
\text { [15,9\%] } \\
\text { 14,7\% mujeres } \\
21,2 \% \text { hombres }\end{array}$ & $\begin{array}{c}\text { Grupo MER } \\
\text { [10\%] } \\
9,8 \% \text { mujeres } \\
11,5 \% \text { hombres }\end{array}$ & $\begin{array}{c}\text { Grupo MAP } \\
{[\mathbf{2 3 , 8 \%}]} \\
24,4 \% \text { mujeres } \\
18,9 \% \text { hombres }\end{array}$ & $\begin{array}{c}\text { Grupo MAP/MER } \\
\text { [15,7\%] } \\
17,1 \% \text { mujeres } \\
9 \% \text { hombres }\end{array}$ & $\begin{array}{c}\text { Grupo MAP/MAR } \\
\text { [23,1\%] } \\
22,6 \% \text { mujeres } \\
27,6 \% \text { hombres }\end{array}$ & $\begin{array}{c}\text { Grupo AMG } \\
{[\mathbf{1 1 , 6 \% ]}} \\
11,4 \% \text { mujeres } \\
11,8 \% \text { hombres }\end{array}$ \\
\hline $\begin{array}{l}\text { Rend. académico } \\
\text { percibido }\end{array}$ & - & - & + & + & + & ++ \\
\hline $\begin{array}{l}\text { Nivel conoci- } \\
\text { mientos }\end{array}$ & - & 一 & + & & + & ++ \\
\hline $\begin{array}{l}\text { Satisfacción con } \\
\text { profesores }\end{array}$ & - & & ++ & & & \\
\hline Valor tareas & - & - & ++ & + & + & + \\
\hline $\begin{array}{l}\text { Creencias de con- } \\
\text { trol }\end{array}$ & - & - & ++ & + & + & + \\
\hline $\begin{array}{l}\text { Creencias de au- } \\
\text { toeficacia }\end{array}$ & - & - & + & & ++ & + \\
\hline $\begin{array}{l}\text { Ansiedad ante los } \\
\text { exámenes }\end{array}$ & & + & - & + & & + \\
\hline \multicolumn{7}{|c|}{$\begin{array}{l}\text { Grupo BMG (baja motivación generalizada), } \mathrm{G} \\
\text { nio de metas de aprendizaje y de metas de evit } \\
\text { motivación generalizada). } \\
++=\text { las puntuaciones más altas en la variable } \\
+=\text { puntuaciones altas en la variable } \\
-=\text { puntuaciones bajas en la variable }\end{array}$} \\
\hline
\end{tabular}


grupo con un perfil motivacional orientado al aprendizaje y a conseguir mejores resultados académicos que los demás (Grupo MAP/MAR), y por último, un sexto grupo con un perfil de alta motivación generalizada (Grupo AMG).

En cuanto a las diferencias entre los grupos (calculadas mediante un MANOVA), el grupo AMG presentaba unas puntuaciones más altas que el resto de los grupos en rendimiento académico percibido y nivel de conocimientos, mientras que el grupo MER presentaba unas puntuaciones más bajas que el resto de los grupos en estas variables (compartidas en algunos casos con el grupo BMG). Además, el grupo MAP era el que mostraba un mayor grado de satisfacción con sus profesores, mientras que el grupo BMG era el que estaba menos satisfecho.

Por tanto, el perfil motivacional donde predominan las tres metas evaluadas (grupo AMG) es, a menos en el contexto universitario, el que presenta unas puntuaciones más altas en cuanto al rendimiento académico percibido y al nivel de conocimientos. De esto se deduce que aquellos universitarios que están motivados para aprender, pero también para conseguir mejores resultados que los demás y para evitar dar una mala imagen ante ellos, son los que informan de un mejor rendimiento académico y los que creen también tener un nivel más alto de conocimientos en las materias académicas que están cursando. Por el contrario, el perfil motivacional orientado a evitar dar una mala imagen ante los demás (grupo MER) es el que obtiene las puntuaciones más bajas en estas variables. El miedo al fracaso, que caracteriza a los estudiantes con este perfil motivacional, los lleva a una constante preocupación por evitar parecer incompetentes ante los demás. Esto, que puede tener sus beneficios para la propia imagen, puede tener consecuencias muy negativas para la implicación y el compromiso del estudiante a nivel académico (Valle et al., en prensa).

Por lo que se refiere a las diferencias en algunas variables motivacionales, el grupo MAP era el que valoraba más las tareas, se sentía más eficaz y con más control sobre el propio proceso de aprendizaje. El grupo MAP, el grupo MAP/MAR y el grupo AMG eran los que mostraban una autoeficacia más alta, mientras que los grupos BMG y MER eran los que presentaban las puntuaciones más bajas.

El Grupo MAP presentaba, por otra parte, los niveles más bajos de ansiedad ante los exámenes, mientras que los grupos donde predominaban, de modo individual o combinado 
con otras, las metas de evitación del rendimiento (grupo MER, grupo MAP/MER y grupo AMG) eran los que mostraban los mayores niveles de ansiedad (una síntesis de estos resultados puede verse en la Tabla 5).

De todas formas, con independencia de las aportaciones de los trabajos mencionados, siguen existiendo dudas en torno a la dinámica de las múltiples metas, lo que hace necesario continuar investigando en esta prometedora línea de trabajo. Parece evidente que la articulación de ambas orientaciones de meta -aprendizaje y rendimiento- implica la puesta en marcha de mecanismos de autorregulación del aprendizaje que permitan una utilización estratégica de las mismas, priorizando una u otra en cada situación particular, dependiendo de los propósitos, motivos o razones para implicarse o no en la tarea de aprendizaje. Esta forma de actuar implica una coordinación y un ajuste efectivo entre los valores y las razones personales y las demandas específicas del contexto de aprendizaje. Un ejemplo propuesto por Paris, Byrnes y Paris (2001) puede resultar esclarecedor respecto a esta idea. Pensemos en un estudiante que está realizando una actividad de matemáticas y que observa cómo otros compañeros terminan la tarea con gran rapidez. Si el estudiante se está encontrando con dificultades para resolver la actividad puede atribuir esta situación a su propia capacidad (en este caso, falta de capacidad para las matemáticas). Esta explicación podría conducir al estudiante a abandonar sus intentos de resolver con éxito la actividad y, en su lugar, copiar la respuesta, lo que le permite dar la imagen de que ha resuelto la tarea con prontitud, aparentando ante los demás que se posee capacidad para resolver la tarea con facilidad. En este ejemplo, el estudiante abandona sus propósitos iniciales de aprender y opta por una meta de aproximación al rendimiento. Así, las condiciones que rodean la realización de las tareas pueden incidir en la forma de concebir la tarea y en el sentido de la propia competencia para resolverlas, lo que quedaría reflejado en la evolución o desarrollo de las orientaciones a meta adoptadas.

La metáfora de los "múltiples caminos" utilizada por Pintrich (2000c) supone un intento de sintetizar las divergencias existentes entre algunas de las investigaciones llevadas a cabo en el campo de las metas académicas. Este autor plantea que las metas de aprendizaje y las metas de aproximación al rendimiento pueden adoptarse y fomentar diferentes patrones de motivación, afecto, uso de estrategias y rendimiento con tiempo. En este sentido, los estudiantes que adoptan distintas metas pueden seguir diversos caminos o trayectorias a lo largo del tiempo, con algunos de ellos finalizando en el mismo lugar en términos de rendimiento real, pero implicando una experiencia muy diferente hasta lograr el resultado final. Así, los estu- 
diantes orientados al aprendizaje vivirían una experiencia "más tranquila y agradable" en términos de motivación, afecto positivo, esfuerzo y uso de estrategias, en su camino hacia unos buenos niveles de rendimiento. Por el contrario, aquellos estudiantes con metas de rendimiento, aun pudiendo alcanzar niveles equivalentes e incluso superiores a los estudiantes con metas de aprendizaje, podrían experimentar menos interés, afecto positivo y tal vez más ansiedad o afecto negativo, dadas sus preocupaciones por superar a los demás. Asimismo, establecido el propósito de parecer más listos que sus compañeros, en contextos "amenazantes" cabe la posibilidad de que el estudiante trate de evidenciar la dedicación de un menor esfuerzo frente a las dificultades o cuando cabe la posibilidad del fracaso a lo largo del camino, lo que supondría costes para el estudiante en términos afectivos (menor interés y más afecto negativo), o les conduciría al empleo de estrategias específicamente dirigidas a lograr esa meta de ser mejores que los demás.

En consecuencia, una de las propuestas más relevantes y con mayor proyección en el propio contexto educativo, en el cual las metas adquieren verdadero sentido y significado, es la de las múltiples metas. Ya el propio Pintrich (2003) estaba firmemente convencido de que muchas de las controversias en torno a las metas debían resolverse con más investigación empírica, contemplando como prioridades en la agenda de la investigación motivacional las siguientes: "La investigación futura sobre metas de logro necesita ir más allá de la diferenciación simplista entre metas de aprendizaje (buenas) versus metas de rendimiento (malas) y considerar las múltiples metas, los múltiple resultados y los múltiples caminos para aprender y rendir en múltiples contextos “ (p. 676).

\section{Metas académicas y motivación: Algunas implicaciones educativas}

Es indudable que conocer los motivos y las metas que llevan a los alumnos a implicarse o no en el aprendizaje no sólo es relevante desde el punto de vista motivacional, sino que también es una cuestión clave para ayudar a comprender mejor los procesos de aprendizaje y las variables que los determinan (Steinmayr y Spinath, 2009). De hecho, una gran parte de la investigación en el campo de las metas académicas se ha centrado en el análisis individual de los distintos tipos de metas y sus relaciones con otras variables relevantes en términos de aprendizaje (p.e., $\mathrm{Ng}, 2008)$.

Sin embargo, esto ha supuesto una visión excesivamente estática e individualista de los motivos y razones que guían la conducta académica de los estudiantes. Es más, este enfo- 
que presenta grandes inconvenientes cuando la propia realidad educativa sugiere que los estudiantes rara vez adoptan una única meta de manera exclusiva, sino que en la mayor parte de los casos, suelen optar por varias metas al mismo tiempo o, dependiendo de las circunstancias, dan prioridad a unas metas sobre otras.

Por eso, como respuesta a cierto estancamiento y a una visión un tanto parcial que se estaba produciendo dentro de la investigación sobre metas académicas, empieza a surgir con fuerza un nuevo enfoque más dinámico y realista que parte del estudiante y de cómo usa diferentes claves motivacionales a nivel personal y contextual para establecer una determinada combinación de metas. Desde esta perspectiva, se contempla la motivación del estudiante como un proceso de gestión de múltiples metas, lo que implica una coordinación y un ajuste efectivo entre los motivos personales y las demandas específicas del contexto de aprendizaje.

Las diferentes combinaciones de metas dan lugar a diferentes perfiles motivacionales definidos en función del mayor o menor peso que tiene cada una de las metas dentro de cada perfil. Por tanto, cada perfil viene a representar a un grupo de estudiantes que usan de modo homogéneo una combinación determinada de motivos, pero además también refleja una manera diferente de estar motivados a nivel académico. A continuación vamos a describir algunas de las principales características de cada uno de estos perfiles motivacionales en aquellas variables consideradas relevantes para el aprendizaje. Además, también se plantean algunas implicaciones educativas derivadas del análisis de cada uno de esos perfiles motivacionales.

- Perfiles motivacionales con preferencia relativa por metas de aprendizaje (por ejemplo, los grupos $M A P, M A P / M A R, M A P / M E R$ y $A M G$ en el estudio de Valle et al., en prensa)

En términos generales los perfiles motivacionales que evidencian en alguna medida la adopción de metas de aprendizaje pueden asociarse a los patrones afectivo-motivacionales y cognitivos más adaptativos. Así, el grupo MAP podría caracterizarse por un alto valor de la tarea y motivación intrínseca, por una alta percepción de control y creencias de autoeficacia. Al tiempo, pueden constituirse en los grupos con la más baja ansiedad académica ante los exámenes, con más baja motivación extrínseca y con baja necesidad de dirigir esfuerzos hacia la autoprotección de la valía. Este perfil integraría a estudiantes con alto empleo de estrategias cognitivas y de estrategias para la autorregulación del propio aprendizaje y con buen rendi- 
miento académico. Sabemos además que se trata de individuos satisfechos con la actuación docente y con un alto nivel percibido de conocimientos. De un modo simplificado podría tratarse de esos estudiantes que disfrutan trabajando.

El perfil MAP/MAR, donde se combinan las metas de aprendizaje y de aproximación al rendimiento, podría presentar un patrón similar al MAP en términos de valor de la tarea, creencias de control y de autoeficacia. Al tiempo que pueden mostrar una motivación extrínseca más importante y una motivación intrínseca menos relevante que el grupo MAP, se observan tasas ansiedad más bajas que para el grupo AMG - con altas motivación generalizada-. Respecto a otros grupos con preferencia por metas de aprendizaje (MAP, AMG, MAP/MER) podría tratarse además del grupo de individuos más activo a la hora de derivar esfuerzos a la protección de la valía en términos de autoafirmación y/o self-handicapping. Estaría integrado por aquellos estudiantes que disfrutan con las tareas académicas y/o contextos académicos de bajo riesgo.

En términos generales, la retroalimentación en el aula, además de contribuir a que los alumnos reconozcan sus virtudes y puedan, por tanto, recurrir a ellas en el futuro, es un recurso que el docente puede emplear para sostener el compromiso de sus estudiantes. Mediante la manipulación de las creencias de control y en la medida que se facilite la asociación del éxito a factores que realmente el estudiante tiene bajo control se facilitará la implicación académica de estos estudiantes. En este punto, el papel del esfuerzo ha recibido especial atención: si los individuos creen que fallaron en el pasado por falta de capacidad, pueden no invertir hoy mucho esfuerzo para tener éxito; sin embargo, si logramos que entiendan que sus fracasos se han debido a su baja dedicación y esfuerzo, tendremos posibilidades de que trabajen más duro, básicamente porque el esfuerzo es una variable que el individuo puede manejar.

Así, desde el punto de vista educativo, será interesante que especialmente este grupo de estudiantes tome conciencia del importante papel que juega el esfuerzo en el aprendizaje. La retroalimentación docente deberá permitir que los buenos resultados y los avances de los aprendices se atribuyan a su dedicación, concentración, perseverancia, etc. Vincular los éxitos a la inteligencia y capacidad puede conseguir que el docente tenga poco que hacer para mantener el compromiso cuando los aprendices orientados al rendimiento, acostumbrados a estar entre los primeros de la clase, dejen de estarlo o cuando simplemente, alguna materia comience a resultarles más difícil. 
Puede ser especialmente adecuado para la intervención motivacional con este perfil crear un clima de aula en el que el fracaso no se considere una amenaza y la capacidad se perciba como un rasgo mejorable a través, precisamente, del esfuerzo. Además, también es importante que los estudiantes con este perfil motivacional tomen conciencia del papel que juegan los mecanismos de autoprotección de la valía y de sus consecuencias sobre el aprendizaje. Así, el docente no deberá permitir los intentos de estos estudiantes de descargarse de la responsabilidad de sus buenos resultados excusándose, por ejemplo, en la facilidad, la suerte o que contaron con ayuda, desestimando su propia actuación, el estudiante evita tener que repetir sus buenos resultados. Al tiempo, debemos ser especialmente prudentes al aceptar disculpas o excusas como la falta de tiempo o el exceso de trabajo o compromisos e incidir en la idea de que los altos niveles de esfuerzo no tienen porque implicar bajos niveles de capacidad.

De los perfiles caracterizados por un predominio relativo de metas de aprendizaje, el grupo MAP/MER, a pesar de manifestar el alto valor de la tarea característico de estos perfiles, es posiblemente el que evidencia un patrón motivacional menos adaptativo en términos de creencias de control y autoeficacia y, de hecho, es de los cuatro grupos con orientación al aprendizaje el más pesimista respecto al nivel de conocimientos adquiridos. A pesar de este patrón pesimista -debido posiblemente al carácter defensivo de la orientación de evitación del rendimiento-, se muestra menos inclinado que el grupo MAP/MAR a derivar esfuerzos a la presentación mejorada de la imagen a través de la autoafirmación y/o el self-handicapping.

Sabemos que los estudiantes con patrones atribucionales más adaptativos suelen creer que los fallos que cometen tienen solución porque dependen de la voluntad de uno o que pueden pensar que los fracasos son ocasionales y se deben a algún factor externo como la dificultad de la tarea o la mala suerte, sin embargo, es posible que un perfil MAP/MER observe sus errores como más inevitables al considerar que se deben a su baja capacidad.

La intervención motivacional con este perfil motivacional debería atender especialmente a la promoción de una creencia de capacidad como una característica variable, modificable y mejorable con la dedicación. Además de procurar la conexión del éxito con el esfuerzo personal, se sugiere la necesidad de proporcionar oportunidades para que el alumno obtenga calificaciones positivas que permitan el feedback docente o el autorrefuerzo. Si transmiti- 
mos mensajes a estos alumnos de modo que entiendan que es el esfuerzo lo que les llevará a mejores resultados estaremos poniendo en sus manos la posibilidad de cambiar sus vidas.

En este caso, el entrenamiento atribucional deberá apoyarse también en la mejora de las estrategias cognitivas y metacognitivas necesarias para abordar con eficacia las tareas escolares. En este sentido, el modelo metacognitivo de Borkowski y Muthukrishna (1992) destaca la importancia de desarrollar y perfeccionar los procesos estratégicos y ejecutivos de aprendizaje, ya que, de esta forma, el individuo podría tomar conciencia de la utilidad e importancia del comportamiento estratégico.

El perfil AMG, con altas metas de aprendizaje pero también altas metas de evitación y de aproximación al rendimiento, se caracterizarían por un valor de la tarea, motivación intrínseca, percepción de control y creencias de autoeficacia similares a los del grupo MAP. Sin embargo, al contrario que los grupos MAP, se trataría de estudiantes con más elevada motivación extrínseca, con una alta tasa de ansiedad académica y que recurren con mayor frecuencia a mecanismos de pesimismo defensivo.

A pesar de mostrar un patron motivacional a priori menos adaptativo, estos estudiantes reconocen un rendimiento académico y un nivel de conocimientos incluso más elevado que el del grupo MAP. El rendimiento de este grupo podría responder más que al empleo de estrategias cognitivas y de autorregulación, dirigidas específicamente al procesamiento significativo de la información, a la gestión activa de recursos de apoyo como el propio esfuerzo y el tiempo. Se trata de estudiantes especialmente preocupados por el rendimiento académico y el nivel de conocimientos, estudiantes que gestionan un complejo de metas diversas. Estos estudiantes que trabajan para aprender pero también para demostrar competencia y evitar mostrar incompetencia, posiblemente deben dedicar importantes esfuerzos a controlar su ansiedad y a gestionar el tiempo y el esfuerzo.

Desde un punto de vista educativo, el docente deberá colaborar con el estudiante ayudándole a disminuir sus tasas de ansiedad y a establecer unos parámetros razonables en torno a las propias expectativas y autoexigencia. Es especialmente importante que estos estudiantes aprendan a reconocer de qué modo sus propias aspiraciones y creencias autorreferidas están detrás de sus reacciones (autoconocimiento afectivo-motivacional) como paso previo 
para llegar a adoptar las estrategias que les permitan enfrentarse a las diferentes situaciones académicas (autocontrol afectivo-motivacional).

La elaboración de listas de situaciones desencadenantes -como por ejemplo la situación de examen o la exposición en público-, la reflexión sobre los indicadores de sentimientos distorsionantes, el análisis de lo que los estudiantes hacen habitualmente para calmarse o para resolver un conflicto, pueden ser actividades instruccionales interesantes. Además, el entrenamiento sistemático en competencia social -en escenarios sociales es donde los estudiantes proyectan gran parte de sus emociones negativas- y la práctica positiva de las estrategias de gestión emocional deberían centrar la atención de la intervención motivacional.

Por otra parte, el docente puede ayudar a estos estudiantes a dirigir su atención hacia el propio proceso de aprendizaje y configurar escenarios educativos menos normativos y menos focalizados en los resultados. Probablemente, la instrucción en estrategias cognitivas y de autorregulación, además de ayudar a estos aprendices a tomar conciencia del proceso de aprendizaje, puede facilitar el establecimiento del vínculo entre el procesamiento profundo y autorregulado y el rendimiento académico. El trabajo en está línea debe permitir el afrontamiento del proceso de aprendizaje de un modo mucho más tranquilo y relajado.

En el ámbito educativo, debe atenderse también a la adopción de mecanismos de pesimismo defensivo o autoprotector en un intento de defenderse de los afectos negativos y, específicamente, de la ansiedad asociada a una deficiente ejecución real o potencial. El mantenimiento de bajas expectativas de éxito poco ajustadas a la realidad ante las tareas puede convertirse en un mecanismo motivacional al que el estudiante acaba recurriendo con objeto de reducir los niveles de ansiedad, al tiempo que la visualización del potencial fracaso futuro puede mantener la dedicación e incluso un aprendizaje de cierta calidad.

- Perfiles motivacionales con preferencia por metas de evitación del rendimiento (por ejemplo, Grupo MER, en el estudio de Valle et al., en prensa)

El grupo MER, con predominio de metas de evitación del rendimiento, puede sostener un patrón de motivación desadaptativo en términos de valor de la tarea, controlabilidad y autoeficacia. Este patrón unido a una alta ansiedad característica de la orientación a metas de 
evitación del rendimiento puede estar detrás de la baja implicación cognitiva que caracteriza al grupo en términos de estrategias cognitivas, estrategias de autorregulación y estrategias de gestión de otros recursos vinculados con el estudio. Puede tratarse además del grupo de estudiantes más proclive a la adopción de mecanismos defensivos y de autoprotección de la valía. Se trata de estudiantes que a pesar de no valorar intrínsecamente la actividad académica sí sostienen razones extrínsecas, instrumentales vinculadas a la imagen. Esta preocupación por la imagen combinada con el hecho de que se trata de estudiantes con una baja percepción de control y autoeficacia en su actividad académica, puede estar detrás de derivar el esfuerzo hacia la protección de la valía en términos de autoafirmación, self-handicapping y pesimismo defensivo.

Además de tomar conciencia de los comportamientos que pueden ser interpretados como formas de protección de la autovalía -aplazamiento de obligaciones, implicación en excesivos proyectos, evitación de responsabilidades, externalización del fracaso, etc.,- y de sus efectos perniciosos sobre el aprendizaje y el rendimiento, desde un punto de vista educativo, estos estudiantes deberían modificar sus creencias respecto a las relaciones esfuerzocapacidad.

Cabe sugerir también la necesidad de evidenciar el valor de logro, intrínseco e instrumental de las actividades y contenidos académicos a los que se enfrenta el estudiante. Además de incrementar la significatividad de los aprendizajes y las posibilidades de autorregulación, la exposición directa de la utilidad para el desarrollo profesional futuro, respecto a otros contenidos temáticos relacionados o como instrumentalmente valioso, respecto a la formación básica como individuo o para la vida diaria, etc., facilitará la labor de mediación docente con este perfil motivacional,

El grupo MER mostrará un característico pesimismo respecto al rendimiento académico y al nivel de conocimientos. Se trata de estudiantes que valoran relativamente las tareas y consideran cierto grado de responsabilidad sobre sus resultados pero sostienen una baja percepción de autoeficacia, considerando además que tienen un mal rendimiento y un nivel limitado de conocimientos, con lo que posiblemente mostrarán una alta ansiedad ante los exámenes. 
Desde un punto de vista educativo, estos estudiantes necesitan incrementar la confianza en sí mismos y en sus posibilidades. A este respecto, algunos estudiantes procuran observar las tareas en trozos, no consideran las actividades como un todo sino como una secuencia de partes, cuando deben abordar conceptos o ideas complejas procuran analizarlos paso a paso, ir comprendiendo cada palabra, cada frase, etc. Esto hace que los retos parezcan más fáciles y alcanzables. Otros buscan una ayuda adaptativa y procuran estudiar con un compañero, van comparando sus notas y sus apuntes con los de los demás, comparan los textos con las notas de clase, o buscan la ayuda del profesor o de otros adultos en aspectos concretos del material de trabajo.

Las pautas de intervención educativa destinadas a la mejora de estas percepciones de competencia se centran en las experiencias de éxito, la búsqueda de conexiones informativas, el desarrollo de la metacognición y de las habilidades de autorregulación. A medida que nuestros aprendices observan la relación entre las nuevas ideas y conceptos y los conceptos e ideas que ya dominan; a medida que observan las similitudes entre el ejercicio o problema que le planteamos y aquellos otros que han abordado en el pasado, la percepción de capacidad para desarrollar ese ejercicio o comprender aquel concepto mejora.

Por otra parte, explicitar con claridad qué se espera del alumno, cuál es el propósito y cuáles pueden ser los caminos para alcanzar los objetivos, hace que estos se consideren más alcanzables. También será preciso que sean más conscientes de la utilización de estrategias de aprendizaje y de sus ventajas y beneficios de cara a mejorar sus resultados académicos. En términos generales, a medida que se potencia el conocimiento metacognitivo del estudiante y se contribuye al desarrollo de sus capacidades de autorregulación se afianza la confianza de los aprendices.

- Perfil motivacional con baja motivación generalizada (Grupo BMG en el estudio de Valle et al., en prensa)

En términos motivacionales, el grupo BMG puede estar integrado por estudiantes que no valoran las tareas de estudio y muestran una baja motivación intrínseca, se muestran poco satisfechos con la labor docente, tienen una baja percepción de control y baja autoeficacia percibida pero, al contrario que los grupos con niveles relativos de orientación al rendimiento, tampoco parecen sostener razones extrínsecas para su implicación en el estudio. Este patrón 
motivacional característico se asocia a una baja implicación en términos de estrategias cognitivas, de autorregulación del proceso de aprendizaje y de gestión de otros recursos. Así, este grupo de estudiantes puede caracterizarse por un bajo nivel de conocimientos percibido y un rendimiento académico bajo. En este grupo se integrarían estudiantes que se perciben poco eficaces y con baja controlabilidad en el entorno académico pero que tampoco valoran la labor académica, ni intrínseca ni instrumentalmente. Se trata de estudiantes que no se complican la vida excesivamente con estrategias de procesamiento cognitivo, de autorregulación ni de gestión pero que tampoco observan especial necesidad de autoprotección de la valía. En realidad, estamos ante aquellos estudiantes que no les interesa trabajar.

Desde un punto de vista educativo, es importante que haya cambios sustanciales en las actitudes educativas de estos estudiantes. Para ello, será necesario trabajar numerosos aspectos vinculados al interés y al valor intrínseco e instrumental de las actividades académicas. Además, todo esto tendrá que ir acompañado de una mejora en las estrategias de aprendizaje y en la toma de conciencia por parte del estudiante de su utilidad en el proceso de aprendizaje.

Tal y como tratamos de sugerir, estas investigaciones sobre los perfiles motivacionales pueden tener importantes implicaciones educativas en la medida que suponen un primer paso en la atención a la diversidad motivacional. De la misma forma que los alumnos son diferentes en sus conocimientos y competencias también son diferentes a nivel motivacional. Asumir diferencias en los perfiles motivacionales de los estudiantes implica que el docente debe partir de los motivos reales del alumno, los cuales son múltiples y diversos.

Esta atención a la diversidad supone por parte del profesor trabajar en esa zona de desarrollo próximo motivacional (Brophy,1998). Además, esta diversidad de motivos también implica que hay una diversidad de caminos desde el punto de vista motivacional para lograr el aprendizaje y el éxito académico. Sí es verdad que algunos son más deseables que otros, pero no todos lo estudiantes tiene por que seguir la misma trayectoria motivacional (Valle et al., 2009).

De este modo, aunque varias orientaciones motivacionales pueden conducir a un mismo resultado, la experiencia real del camino recorrido suele ser muy diferente de unos casos a otros. Así, en algunas circunstancias esa experiencia es mucho más tranquila y agradable, mientras que en otros se convierte en un camino de tensiones y sobresaltos. Por decirlo de 
otra manera, en ocasiones esa trayectoria motivacional es como viajar por una autopista, con poco tráfico y con buenas condiciones meteorológicas, mientras que en otras se convierte en un viaje mucho más lento, por una carretera con muchas curvas, con mucho tráfico y numerosos riesgos para el conductor.

Que el profesor sea capaz de adaptar sus recursos y actividades instruccionales a los múltiples itinerarios motivacionales de los alumnos es una de las claves para garantizar unos buenos resultados desde el punto de vista motivacional.

Por eso, de cara a la investigación futura es necesario profundizar en el papel del docente como verdadero agente motivador de los estudiantes (Gonida, Voulala y Kiosseoglou, 2009), y de cómo sus actividades pueden contribuir a que se desarrollen en el aula patrones motivacionales adaptativos. Sin duda que el trabajo que se realice con el profesorado para lograr su implicación será una de las claves para conseguir avances importantes en este tipo de investigaciones.

\section{Nota}

Este trabajo forma parte de otras investigaciones más amplias que han sido financiadas por la DGI-MEC (SEJ2006-01518) y por la DGU-MEC (EA2007-0242). 


\section{Referencias}

Atkinson, J.W. (1957). Motivational determinants of risk-taking behavior. Psychological Review, 64, 359-372.

Ames, C. (1984). Competitive, cooperative, and individualistic goal structures: A motivational analysis. En R. Ames y C. Ames (Eds.), Research on motivation in education: Vol 1. Student motivation (pp. 177-208). New York: Academic Press.

Barron, K.E., y Harackiewicz, J.M. (2001). Achievement goals and optimal motivation: Testing multiple goals models. Journal of Personality and Social Psychology, 80, 706722 .

Bartels, J.M., Magun-Jackson, S. y Kemp, A.D. (2009). La regulación volicional y la autorregulación del aprendizaje: un estudio de las diferencias individuales en la motivación de logro aproximación-evitación. Electronic Journal of Research in Educational Psychology, 18, 7(2), 605-626.

Borkowski, J.G. y Muthukrishna, N. (1992). Moving metacognition into the classroom: Working models and effective strategy teaching. En M.Pressley, K.R. Harris y J.T. Guthrie (Eds.), Promoting academic competence and literacy in school (pp. 477-501). San Diego: Academic Press.

Bouffard, T., Boisvert, J., Vezeau, C. y Larouche, C. (1995). The impact of goal orientation on self-regulation and performance among college students. British Journal of Educational Psychology, 65, 317-329.

Brophy, J. (1998). Motivating students to learn. Nueva York: Mc Graw-Hill.

Cabanach, R.G., Valle, A., Piñeiro, I., Rodríguez, S. y Núñez, J.C. (1999). El ajuste de los estudiantes con múltiples metas a variables significativas del contexto académico. Psicothema, 11, 313-323.

Daniels, L.M., Haynes, T.L., Stupnisky, R.H., Perry, R.P., Newall, N.E. y Pekrun, R. (2008). Individual differences in achievement goals: A longitudinal study of cognitive, emotional, and achievement outcomes. Contemporary Educational Psychology, 33, 584608 .

Dweck, C.S. (1986). Motivational processes affecting learning. American Psychologist, 41, 1040-1048.

Elliot, A. (1997). Integrating the "classic" and "contemporary" approaches to achievement motivation: A hierarchical model of approach and avoidance achievement motivation. En M.L. Maehr y P.R. Pintrich (Eds.), Advances in motivation and achievement (pp. 143-179). Greenwich, CT: JAI. 
Elliot, A.J. (1999). Approach and avoidance motivation and achievement goals. Educational Psychologist, 34, 169-189.

Elliot, A.J. y Church, M.A. (1997). A hierarchical model of approach and avoidance achievement motivation. Journal of Personality and Social Psychology, 72, 218-232.

Elliot, A.J. y Harackiewicz, J.M. (1996). Approach and avoidance achievement goals and intrinsic motivation: A mediational analysis. Journal of Personality and Social Psychology, 70, 461-475.

Elliot, A.J. y McGregor, H.A. (2001). A 2x2 achievement goal framework. Journal of Personality and Social Psychology, 80, 501-519.

Elliot, A.J., McGregor, H.A. y Gable, S. (1999). Achievement goals, study strategies, and exam performance: A mediational analysis. Journal of Educational Psychology, 91, 549-563.

Gonida, E. N., Voulala, K. y Kiosseoglou, G. (2009). Students' achievement goal orientations and their behavioral and emotional engagement: Co-examining the role of perceived school goal structures and parent goals during adolescence. Learning and Individual Differences, 19, 53-60.

Harackiewicz, J.M., Barron, K.E., Pintrich, P.R., Elliot, A. J. y Thrash, T.M. (2002). Revision of achievement goal theory: Necessary and illuminating. Journal of Educational Psychology, 94, 638- 645.

Harackiewicz, J.M., Barron, K.E., Tauer, J.M., Carter, S.M. y Elliot, A.J. (2000). Short-term and long-term consequences of achievement goals: predicting interest and performance over time. Journal of Educational Psychology, 92, 316-330.

Harackiewicz, J.M. y Linnenbrik, E.A. (2005). Multiple achievement goals and multiple pathways for learning: The agenda and impact of Paul R. Pintrich. Educational Psychologist, 40, 75-84.

Levy-Tossman, I., Kaplan, A. y Assor, A. (2007). Academic goal orientations, multiple goal profiles, and friendship intimacy among early adolescents. Cntemporary Educational Psychology, 32, 231-252.

McClelland, D.C. (1951). Personality. Nueva York: The Dryden Press.

Meece, J. y Holt, K. (1993). A pattern analysis of students' achievement goals. Journal of Educational Psychology, 85, 582- 590.

Middleton, M. y Midgley, C. (1997). Avoiding the demonstration of lack of ability: An unexplored aspect of goal theory. Journal of Educational Psychology, 89, 710- 718. 
Ng, Ch.C. (2008). Multiple-goal learners and their differential patterns of learning. Educational Psychology, 28, 439-456.

Nichols, J.G. (1984). Achievement motivation: Conceptions of ability, subjective experience, task choice, and performance. Psychological Review, 91, 328-346.

Pajares, F., Britner, S.L. y Valiente, G. (2000). Relation between achievement goals and selfbeliefs of middle school students in writing and science. Contemporary Educational Psychology, 25, 406-422.

Paris, S.G., Byrnes, J.P. y Paris, A.H. (2001). Constructing theories, identities, and actions of self- regulated learners. En B.J. Zimmerman y D.H. Schunk (Eds.), Self- regulated learning and academic achievement: Theoretical perspectives ( $2^{\mathrm{a}} \mathrm{ed}$.) (pp. 253-288). Mahwah, NJ: Lawrence Erlbaum.

Paris, S.G. y Turner, J.C. (1994). Situated motivation. En P.R. Pintrich, D. Brown y C. Weinstein (Eds.), Student motivation, cognition, and learning: Essays in honor of Wilbert J. McKeachie (pp. 213-237). Hillsdale, NJ: Erlbaum.

Phan, H.P. (2008). Metas de logro, el entorno del aula, y el pensamiento reflexivo: Un marco conceptual. Electronic Journal of Research in Educational Psychology, 6(3), 571-602.

Pintrich, P.R. (2000a). The role of goal orientation in self-regulated learning. En M. Boekaerts, P.R. Pintrich y M. Zeidner (Eds.), Handbook of self-regulation (pp. 451502). San Diego, CA: Academic Press.

Pintrich, P.R. (2000b). An achievement goal theory perspective on issues in motivation terminology, theory, and research. Contemporary Educational Psychology, 25, 92-104.

Pintrich, P.R. (2000c). Multiple goals, multiple pathways: The role of goal orientation in learning and achievement. Journal of Educational Psychology, 92, 544-555.

Pintrich, P.R. (2003). A motivational science perspective on the role of student motivation in learning and teaching contexts. Journal of Educational Psychology, 95, 667-686.

Pintrich, P.R. y Garcia, T. (1991). Student goal orientation and self- regulation in the collage classroom. En M.L. Maehr y P.R. Pintrich (Eds.), Advances in motivation and achievement: Goals and self-regulatory processes (Vol. 7, pp. 371-402). Greenwich, CT: JAI Press.

Rodríguez, S., Cabanach, R.G., Piñeiro, I., Valle, A., Núñez, J.C. y González-Pienda, J.A. (2001). Metas de aproximación, metas de evitación y múltiples metas académicas. Psicothema, 13, 546-550.

Rodríguez, S., Cabanach, R.G., Valle, A., Núñez, J.C. y González-Pienda, J.A. (2004). Diferencias en el uso del self-handicapping y pesimismo defensivo y sus relaciones con las metas 
de logro, la autoestima y las estrategias de autorregulación del aprendizaje. Psicothema, $16,626-632$.

Skaalvik, E. (1997). Self- enhancing and self-defeating ego orientation: Relations with task and avoidance orientation, achievement, self- perceptions, and anxiety. Journal of Educational Psychology, 89, 71- 81.

Steinmayr, R. y Spinath, B. (2009). The importance of motivation as a predictor of school achievement. Learning and Individual Differences, 19, 80-90.

Suárez, J.M., Cabanach, R.G. y Valle, A. (2001). Multiple-goal pursuit and its relation to cognitive, self-regulatory, and motivational strategies. British Journal of Educational Psychology, 71, 561- 572.

Tapola, A. y Niemivirta, M. (2008). The role of achievement goal orientations in students' perceptions of and preferences for classroom environment. British Journal of Educational Psychology, 78, 291-312.

Valle, A., Cabanach, R.G., Núñez, J.C., González-Pienda, J.A., Rodríguez, S. y Piñeiro, I. (2003). Multiple goals, motivation and academic learning. British Journal of Educational Psychology, 73, 71-87.

Valle, A., Cabanach, R.G., Rodríguez, S., Núñez, J.C. y González-Pienda, J.A. (2005). Selfworth protection strategies in higher educational students: Exploring a model of predictors and consequences. En R. Nata (Ed.), New directions in higher education (pp. 99126). Nueva York: Nova Science Publishers, Inc.

Valle, A., Cabanach, R.G., Rodríguez, S., Núñez, J.C. y González-Pienda, J.A. (2006) Metas académicas, estrategias cognitivas y estrategias de autorregulación del estudio. Psicothema, 18, 165-170.

Valle, A., Núñez, J. C., Cabanach, R. G., Rodríguez, S., González-Pienda, J. A. y Rosário, P. (2007). Metas académicas y estrategias motivacionales de autoprotección. Electronic Journal of Research in Educational Psychology, 13, 617-632.

Valle, A., Núñez, J. C., Cabanach, R. G., Rodríguez, S., González-Pienda, J. A. y Rosário, P. (2009). Perfiles motivacionales en estudiantes de Secundaria: Análisis diferencial en estrategias cognitivas, estrategias de autorregulación y rendimiento académico. Revista Mexicana de Psicología, 26, 113-124.

Valle, A., Núñez, J.C., Cabanach, R.G., González-Pienda, J.A., Rodríguez, S., Rosário, P., Muñoz-Cadavid, M. y Cerezo, R. (2009b). Academic goals and learning quality in higher education students. The Spanish Journal of Psychology, 12, 96-105. 
Valle, A., Núñez, J. C., Rodríguez, S., Cabanach, R. G., González-Pienda, J. A. y Rosário, P. (en prensa). Perfiles motivacionales y diferencias en variables afectivas, motivacionales y de logro. Universitas Psychologica.

Was, C., Al-Harthy, I., Stack-Oden, M. y Isaacson, R.M. (2009). Rango de identidad académica y su relación con la orientación de meta de logro. Electronic Journal of Research in Educational Psychology, 18, 7(2), 627-652.

Wentzel, K.R. (2000). What is it that I'm trying to achieve? Classroom goals from a content perspective. Contemporary Educational Psychology, 25, 105-115.

Wentzel, K.R. (2001). Metas socials y relaciones socials como motivadores de la adaptación a la escuela: un análisis motivacional. En J. Juvonen y K.R. Wentzel (Eds), Motivación y adaptación escolar (p. 269-294). México: Oxford University Press.

Wolters, C.A., Yu, S.L., y Pintrich, P.R. (1996). The relation between goal orientation and students' motivational beliefs and self- regulated learning. Learning and Individual Differences, 8, 211- 238. 